\title{
Computational Modeling of Stem Cell Migration: A Mini Review
}

\author{
Xiaofeng Liu, ${ }^{1,2}$ Diego A. Vargas, ${ }^{3}$ Dongyuan Lü, ${ }^{1,2}$ Yan Zhang, ${ }^{1,2}$ Muhammad H. Zaman, ${ }^{3}$ \\ and Mian LONG ${ }^{1,2}$ \\ ${ }^{1}$ Key Laboratory of Microgravity (National Microgravity Laboratory), Institute of Mechanics, Chinese Academy of \\ Sciences, Beijing 100190, China; ${ }^{2}$ Center of Biomechanics and Bioengineering, Institute of Mechanics, Chinese Academy of \\ Sciences, Beijing 100190, China; and ${ }^{3}$ Departments of Biomedical Engineering and Medicine, Boston University, Boston, \\ MA 02215, USA
}

(Received 28 January 2014; accepted 30 March 2014; published online 24 April 2014)

Associate Editor Michael R. King oversaw the review of this article.

\begin{abstract}
Stem cell migration is crucial in many biological processes such as embryogenesis, histogenesis, and stem cell biology. It is also an important process in physiology, and in medicine, it can contribute to develop effective stem cell therapies. While there is a large body of experimental evidence that attempts to understand the biological and physiological mechanisms governing stem cell activity in the organism, computational modeling studies are scarce. Because stem cell migration is affected by biological diversity and the complexity of the cells' microenvironment, experiments are hard to conduct and corresponding measurements are sophisticated. Computational modeling is a good complementary method to help us understand this process. Here, a mini-review is presented discussing the existing efforts and the unsolved key issues in stem cell migration. In addition, existing computational models studying the migration of differentiated cells are briefly discussed in the context of how they can be applied to increase our understanding of the dynamics involved in stem cell migration: Particular emphasis is placed on how biomechanical aspects of migration are explored in these models.
\end{abstract}

Keywords-Computational modeling, Stem cell, Migration, Dynamics.

\section{INTRODUCTION}

In past decades, it has been suggested that cell migration is one of the most important biological processes; it is crucial to immune responses, tumor metastasis, wound healing, and histogenesis. This is

Address correspondence to Muhammad H. Zaman, Departments of Biomedical Engineering and Medicine, Boston University, Boston, MA 02215, USA and Mian Long, Key Laboratory of Microgravity (National Microgravity Laboratory), Institute of Mechanics, Chinese Academy of Sciences, Beijing 100190, China. Electronic mail: zaman@bu.edu,mlong@imech.ac.cn

Xiaofeng Liu, Diego Vargas, and Dongyuan Lü contributed equally to this work. supported by a body of evidence from experimental measurements, as well as computational models. The study of biological systems through computational modeling yields some of the following advantages: (1) models allow for easy quantification of elements that are technically difficult to measure experimentally, (2) they allow us to carry out experiments that are financially prohibitive, and (3) they can be used to design future experiments and predict their outcome. Specifically, computational modeling is widely applied in the study of mechanosensing and mechanotransduction in cell migration; it has been used to characterize the resulting phenomena such as cell mechanotaxis, ${ }^{52}$ durotaxis, ${ }^{39}$ and tensotaxis. ${ }^{4,6}$ Similarly, it has been used to understand phenomena resulting from chemically induced migration, such as haptotaxis ${ }^{59}$ and chemotaxis. ${ }^{17}$ In this review, we focus on computational modeling of stem cell migration. Efforts modeling of other cell types are shortly outlined and their potential application in the study of stem cells discussed.

\section{IMPORTANCE OF STEM CELL MIGRATION IN PHYSIOLOGY AND MEDICINE}

Different stem cell populations have been characterized in different biological processes, and their migration is critical during histogenesis, organogenesis, embryonic body formation, tissue homeostasis, and regeneration of organ function. During embryonic development, embryonic stem cells (ESCs) present coordinated migration and programmed differentiation. In early development, the formation of functional organs depends largely on the migration of ESCs from the site of their specification to the target region related to the development of the organ. Hematopoietic 
ontogeny is characterized by a spatiotemporal migration and distribution of hematopoietic stem cells (HSCs), the best characterized adult type of migrating stem cells, during embryogenesis. After embryonic development, during adult life, stem cells mostly migrate in response to tissue regeneration. It is believed that tissue regeneration, via stem cell migration and differentiation to a site of tissue injury, is not limited to the hematopoietic system. Thus, stem cell migration contributes greatly to understand the basic principles in the fields of development and regeneration.

In addition to their role in physiology, stem cells have been suggested to be the source of a small population of cancer cells, referred to as cancer stem cells (CSCs). Metastatic/migrating CSCs are assumed to play a critical role in the dissemination of the disease. In this way the study of stem cells contributes to the understanding of cancer. For this and other reasons stem cell migration is also relevant to clinical medicine. One example is the application of mesenchymal stem cells (MSCs) in regenerative medicine. ${ }^{18}$ Recent studies have demonstrated that MSCs have the ability to aggregate at the site of tissue or organ damage and differentiate into multiple cell types of the mesenchymal lineage, ${ }^{31,48,56,57}$ suggesting great potential in their application to gene therapy and the treatment of heart, brain, and necrotic limb disease. ${ }^{1,10,33}$ It is the migratory potential of stem cells that allows for their use in therapeutics: Implanted MSCs are able to migrate to the injury site, suggesting that an innate response to the injured microenvironment may produce attractive signals such as cytokines and chemokines to recruit those MSCs. ${ }^{26,42}$ The underlying mechanisms that mediate these responses, however, are still unclear in part due to the methodological limitations. Similarly, in the case of HSCs, transplantation is used in treatment of haematological tumors and diseases. The success of this therapeutic procedure is dependent partly on the display of rapid migratory behavior to enter and exit their niche in the bone marrow. Though promising, we are still far away from addressing the resulting issues in HSC migration and differentiation. ${ }^{36,48}$ Understanding stem cell migration will help us optimize target migration, predict the effect of various treatments on specific blood diseases, shorten the period in which the patient's effective immune system is suppressed, and ultimately develop better therapeutics. ${ }^{36,48}$

\section{NEED FOR COMPUTATIONAL MODELING OF STEM CELL MIGRATION}

The aforementioned physiological and clinical importance of stem cell migration justifies the need for a quantitative understanding of (adult) stem cell migration; however, gaining this understanding is still difficult. This is due to the low frequency of these cells in vivo, ${ }^{54}$ the difficulties in identifying and prospectively purifying tissue-specific stem cells near homogeneity, ${ }^{45}$ and, most importantly, the lack of adequate technologies and protocols to study stem cell migration in vivo. Current discoveries in the study of stem cell migration are based mostly on experimental measurements and clinical observations. These experiments attempt to elucidate from statistical results the processes involved in migration, including cell signaling and protein interactions, as well as the effect of exposure to drugs. ${ }^{17,47}$ The evidence collected, however, is insufficient to quantify the stem cell migration dynamics even at single cell level due to the complexity of the issues and the diversity of biological mechanisms present in stem cells. Figure 1 shows the abundance of factors accounting for the complexity of the process of stem cell migration; it summarizes schematically the different chemical factors involved in determining cellular behavior and zooms in on the geometrical and mechanical properties that affect migration for ECMcell interactions (Fig. 1). ${ }^{41}$

To understand the complicated mechanisms of stem cell migration, computational modeling $(\mathrm{CM})$ has become in recent years an important approach to complement existing experimental methods. Building on existing experimental techniques, $\mathrm{CM}$ can incorporate experimental findings and generate predictions

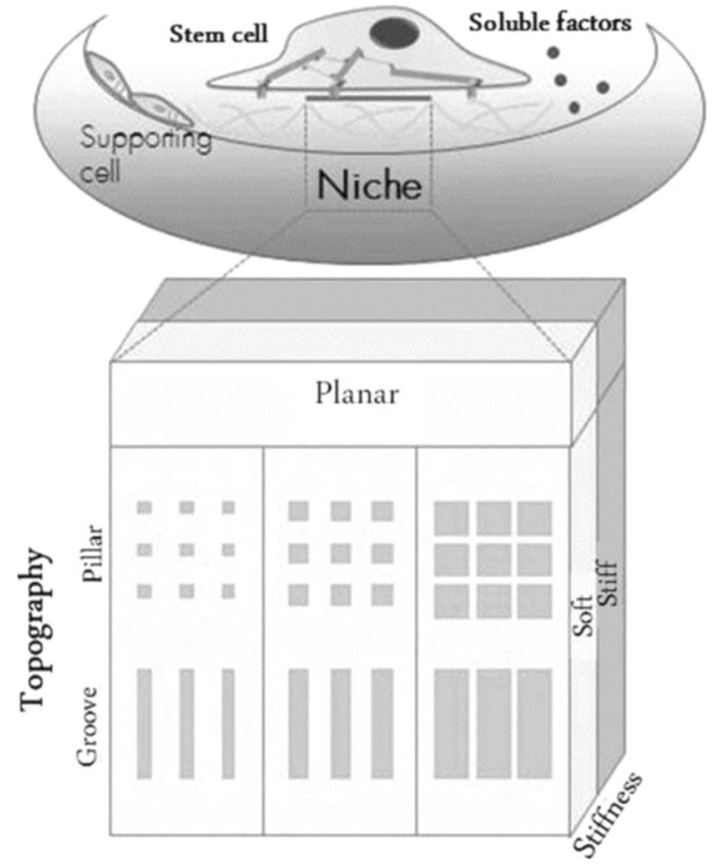

FIGURE 1. Schematic of stem cell migration in a complicated niche. The image shows the different chemical factors (top) and physical properties of ECM-cell interactions (bottom) involved in determining stem cell behavior. Modified from. ${ }^{37}$ 
that can be translated into hypotheses to theoretically quantify the migration dynamics of stem cells. In addition, $\mathrm{CM}$ can make these predictions in a methodical and an efficient manner. It can address questions involving embryonic, hematopoietic, mesenchymal, and cancer stem cells during development and adulthood with a clear emphasis on in vivo conditions. ${ }^{5,27,44,46}$ These computational works pursue reasonable models to mimic the distinct migration dynamics in local microenvironment in single cells or small tissue samples. ${ }^{5,27,44,46}$ In some of these, numerical simulations can provide us with access to those unknown aspects of cell behaviors and tissue functions: Models that account for key biological regulators can formulate predictions that are hard to make from collected results from specific experiments or statistical data. Moving forward these novel approaches should incorporate interdisciplinary dialogs that coordinate biological tests, mathematical modeling, and computational simulations to validate experimental data and non-intuitive scenarios for stem cell migration.

Even though CM studies on MSC migration remain scant, one existing model displays the potential for $\mathrm{CM}$ in studying MSCs: Modeling has led to an increased understanding of both the engraftment process of stem cells into injury site and the mobilization of stem cells into the peripheral circulation, as compared to numerous experimental studies on MSCs migration to the site of tissue damage. ${ }^{35,38}$ This might shed light on understanding the migration dynamics and clinical issues involved in MSCs transplantation. ${ }^{18}$ In a therapeutic context, the most critical part of MSC transplantation is to devise a mechanism to enhance the number of transplanted cells that migrate to the site of tissue damage.

\section{MAJOR ACHIEVEMENTS IN COMPUTATIONAL MODELS OF STEM CELL MIGRATION}

Stem cells, as a specialized and pluripotent type of cells that differ from differentiated cells in nature, have also attracted the attention of biophysicists and biomechanicians. CM of their migration is of great value to predict their function and to interpret experimental data. To date, only a few mechanical or computational models appear in this regard, as summarized in Table 1, largely due to lack of comprehensive molecular understanding and only recent investigations in the mechanobiology of stem cell differentiation.

$\mathrm{CM}$ of stem cell migration helps to elucidate the spatiotemporal organization of cells during tissue formation. An individual mechanistic cell-based model was proposed to quantitatively describe the spatiotemporal organization of MSCs cultured in a plain or grooved substrate. ${ }^{27}$ Starting from the assumption that the cell dynamics are determined by attractive and repulsive interaction forces between cells and between cells and the substrate, the model employs pseudopodia-generated forces for cell movement and adjusts cell behavior in response to cell density. This model makes a substantial improvement with respect to previous models by explicitly representing cellular podia and their dynamics. Existing podia generate protrusion and traction forces for MSC cell spreading and movement. They are randomly elongated, inactivated, and retract as a result of the effect of their inherent contraction force until the disappearance of those sufficiently retracted podia. The adaptation of podium generation and the probability of inactivation dynamically control the number of podia which in turn determines the migration phenotype of MSCs. The maximum speed of podium migration is governed by the ratio of protrusion force and substrate friction. As a simple, sufficient model, the predicted podium traction dynamics are in agreement with experimental observations for thousands of MSCs on stiff substrate. Another model, also looks at agent-based dynamics but considers the dynamics of multiple cells during tissue organization, specifically the organization of murine intestinal crypts. ${ }^{5}$ Similar to the model discussed previously, this model looks at the effect of regulatory mechanisms in a single cell in migration; however, instead of looking at the protrusion and traction forces experienced by the cells, this model uses interaction energy to quantify cell-cell and cell-basal membrane interactions. The model focuses on a biochemical factor in particular,

TABLE 1. Summary of models of stem cell processes.

\begin{tabular}{lllll}
\hline Stem cell type & \multicolumn{1}{c}{ Model } & Scale & \multicolumn{1}{c}{ Biological processes } & \multicolumn{1}{c}{ References } \\
\hline MSC & Mechanistic & Cell & Division & Harrison et al. $^{25}$ \\
SC in crypts & Dynamic & Tissue & Proliferation, differentiation & Gerisch and Chaplain $^{22}$ \\
MSC & Mechanistic & Tissue & Tissue differentiation & Jiao and Torquato $^{29}$ \\
MSC & Mechanistic, biological & Tissue & Angiogenesis, osteogenesis & Khayyeri et al. $^{30}$ \\
MSC & Biological & Tissue & Proliferation, differentiation, osteogenesis & Kolega $^{32}$ \\
Tumor SC & Dynamic & Cell & Tumor growth & Kotobuki et al. $^{33}$ \\
Plant SC & Biological & Cell & Segmentation, self-renewal, differentiation & Lauffenburger and Horwitz ${ }^{34}$ \\
\hline
\end{tabular}


Wnt-activity. This factor is typically linked to a biomechanical response of tissue; it is linked to the local curvature of the basal membrane as a function of the crypt axis. By integrating different scales of biological architecture (molecular, cellular, and tissue levels), this model is able to account for spatially confined cell proliferation, directed cell migration, multiple cell lineage decision, and clonal competition. The authors arrive at the conclusion that the tissue should be able to fully recover after complete elimination of cellular subpopulations including subpopulations deemed to be functional stem cells. This model is a clear example that a quantitative and dynamic ${ }^{28}$ understanding of the spatial and temporal organization of gene expression and cell behavioral patterns within multilayered and actively growing developmental fields is crucial to model the process of development.

Other models demonstrate further that both mechanical and biological factors play an important role in stem cell migration. A mechanoregulation theory for MSC differentiation and migration was implemented to predict tissue differentiation inside mechanically controlled bone chambers inserted into rat tibae. ${ }^{30}$ MSC migration was executed using a random walk theory; simulations are performed using a stochastic lattice model and a finite element analysis. Direct comparison with experimental data was achieved by simulating an in vivo bone chamber experiment. Regardless of the ability to make direct comparisons between simulated and experimental results, questions on stem cell dynamics remain to be addressed through CM. In this system particularly, questions remain about the link between tissue differentiation and genetic variability in animal populations. Another example of a model integrating both mechanical and biological factors looks at the difference in healing between normal and impaired fractures. $^{21}$ In it, MSC migration, proliferation, differentiation, and growth factor and matrix synthesis were formulated to be dependent on local matrix density and/or growth factor concentration. A highly coupled system of nonlinear partial differential equations (PDEs) is used to mathematically describe the sytem: This system is used to predict the time-dependent chemotaxis or haptotaxis in angiogenesis and osteogenesis. The simulated results confirmed the hypotheses of several experimental studies indicating that suggested an interconnection between angiogenesis and osteogenesis.

When applying CM to the study of stem cells, models should address the major differences in migration dynamics between stem cells and other cells. In general, this difference lies in the unique capacity stem cells have to differentiate. Two different models, an agent-based hybrid model as well as a caricature continuous model, have been used elucidate the progressive development of post-cranial structures from a pool of caudal undifferentiated cells during embryonic axis elongation. The FGF-8-induced chemotaxis that characterizes caudal undifferentiated cells was predicted using an equation for chemotactic energy (hybrid model) or a diffusive gradient. ${ }^{25}$ This model has the potential to be extended to understand other developmental processes displaying a similar mode of axis extension coupled to cell differentiation. Another example of a model taking into account differentiation quantifies diverse aspects involved in the progression of bone healing for moderate fracture gap sizes and fracture stability. ${ }^{3}$ This two-dimensional mathematical model takes into account the impact of concentration or gradient of chemokines and cytokines on multipotential stem cells that differentiate into specialized cells; this model applies haptotactic and haptokinetic cell migration speed to estimate spatiotemporally the rate change of cellular densities for mesenchymal cell migration, mesenchymal cell and chondrocyte/osteoblast proliferation and differentiation, and ECM synthesis and degradation during bone tissue regeneration.

In the study of cancer, models have addressed the differences between mortal tumor cells and tumor stem cells. A particular example focuses on the cytokine gradient-directed migration of a tumor consisting of both types of cancer cells. ${ }^{16}$ An agent-based model mimics tumor migration in two ways: one is directed migration towards an attractor source and the other is random motility without any attractor sources. In the former case, when an exponentially-decayed cytokine gradient is generated with respect to the distance from the attractor source, the tumor cell migration can be divided into two cases: In the first case, tumor cells display directed migration along the gradient until the cells slow down when they reach the source. In the second case, tumor stem cells are initially at the source, and the continuous inbound migration of cells on the periphery happens when they proliferate sufficiently. In the latter case, an interesting find was that as cell crowding inhibits cancer cell proliferation, stem cells are liberated by random cell motility and death among their non-stem progeny, allowing the continual seeding of clones in the tumor periphery that contribute to the efficient expansion of the tumor.

Finally, it is important to note that these achievements in stem cell CM have required a whole array of computational tools in data integration, image analysis, statistical modeling, pattern recognition, machine learning, and dynamical system identification. Two examples of these greater efforts a diverse methodology are listed next: One example is seen in the study of the pattern formation of Arabidopsis shoot apical meristem 
stem cell niche. In this effort, Softassign Procrustes algorithm was employed to solve a point matching problem and to improve the level-set-based tracking method in 3D imaging of cell division and migrating trajectories. ${ }^{7}$ The other example consists of a comprehensive integration performed in a two-tiered visual manner to predict the maturation of HSCs into T cells in the thymus. ${ }^{13}$ This required diverse data sets spanning data from cell migration, cell differentiation, histology, electron microscopy, molecular biology, and biochemistry.

\section{COMPUTATIONAL MODELS PERTAINING TO DIFFERENTIATED CELL MIGRATION}

In contrast to the insufficient modeling for stem cell migration, a vast body of models has been proposed for the migration of differentiated cells. Cell migration is crucial to the understanding of the normal functioning of an organism as well as pathological conditions, and evidence for both single and collective migration has been collected in vivo and in vitro for decades. $^{32}$ And while both the biophysical and molecular mechanisms for single cell migration are relatively well understood, ${ }^{34}$ collective migration has been difficult to characterize because it is inherently a multiscale phenomenon and occurs at a slower time scale than single cell migration. In these aspects, some challenges involved in the modeling of stem cell migration are shared by modeling of collective migration. Additionally, collective migration plays a role in physiological processes that involve stem cells as are tissue repair, morphogenesis, and cancer invasion. ${ }^{20} \mathrm{In}$ effect, many of these models were developed to study cancer. ${ }^{22,29,43}$ Next we review some of the main considerations that have been taken into account in modeling cell migration and briefly discuss how they may be beneficial to improve the modeling of stem cell migration.

The different approaches to modeling cell migration either create a biomechanistic representation of the cell to generate an effective system behavior (i.e. migratory speed and direction), or in contrast, replicate the behavior of the whole cell to make conjectures on the mechanisms inside them. Because the molecular mechanisms behind single migration are understood, models addressing single cell migration tend to be detailed at the molecular scale and have now moved on to look at dynamics at the subcellular scale.

Initial models of single cell migration were based on the dynamics of three major phases of motion or a migration cycle observed in mesenchymal cells. ${ }^{11,58}$ In one cycle, there are three types of force corresponding to different phases: a contractile force from cell membrane protrusions (lamellipodia), a traction force in the front and rear of the cell due to receptor-ligand pairs (focal adhesions), and a resistant force from viscoelastic nature of the ECM. ${ }^{58}$ The entire process is repeated until the cell stops completely on physicochemical constraints. Such models included the effects of both the cytoskeletal contractions and the extracellular environment. Currently, single cell models have expanded to include both time and spatial dynamics within the cell as well as the effect of biochemical rates of intracellular signaling networks in migration. This can be witnessed in both cellular Potts models (CPM) and spatial stochastic models taking into account diffusion and advection within the cytoplasm. ${ }^{9,49,53}$ Other models have been developed studying not the effect of dynamics inside the cell but outside of it: Cell-ECM interactions are crucial to regulation of migration dynamics of single cells. Researchers have considered the effect of contact guidance and matrix remodeling, taking into account matrix stiffness and architecture. $^{24,51,52}$

Through experimental studies of cell migration, it has become apparent that dimensionality of the cellular environment affects cellular behavior and migration mode. Cells will display different behavior when on a one, two or three dimensional space ${ }^{14}$; for example, while most cells have lower speeds in a 3D environment than on a 2D culture surface, neutrophils display almost no migration in 2D substrates and do migrate when embedded in 3D collagen gels. ${ }^{23,24}$ Observations such as this have led to both the development and engineering of in vitro platforms to study migration as well as consideration of this phenomenon in CM. ${ }^{24,49,55}$ The internal differences in gene and protein expression of different cell types are now considered in CM.

This understanding of protein expression and signaling networks in migratory cells have contributed in the initial efforts to model migration of cell collectives. Although collective migration is still being understood, it has been observed to occur through different mechanisms: vascular sprouting for angiogenesis, branching for morphogenesis in mammary glands, free groups of border cells, detached clusters, and multicellular 3D invasion strands in cancer. ${ }^{19}$ Derived from forced based mechanistic models of single cell migration, a model of a cell cluster was developed taking into consideration the impact of three major factors: ligand concentration, matrix metalloproteinase activity, and cluster geometry. ${ }^{55}$ This model is promising, successfully showing potential effects of altering single variables in a system of cells in motion.

As a last consideration, we will mention tumor growth computational models. As stated above, many collective cell migration models were developed to 
study cancer, and one category of these is that simulating tumor growth. These models focus on finding the influence of external cues such as oxygen and nutrient availability on the shape and compactness of growing cell masses. They can be found both as agentbased models or treating cell populations as a unit as in continuum models. ${ }^{2,12,50}$ Agent-based models allow for modeling of different cell types in populations; this can be utilized when studying the role of stem cells in tumor metastasis and histogenesis.

\section{FUTURE PERSPECTIVES}

Computational modeling (CM) has emerged as a novel and highly promising strategic approach in various fields of biomedical sciences, particularly when the experimental approach or clinical investigation is technically challenging or expensive: It is important to emphasize that the mechanical microenvironment of stem cells, which largely governs their migration dynamics, is quite complicated in vivo (Fig. 2) ${ }^{40}$ While a few models have been proposed to illustrate theoretically or numerically the migration dynamics, there are, at least, the following aspects to be addressed through CM:

1. At the tissue level, CM can be used to understand how stem cells migrate in the cases of embryonic development and tissue histogenesis because many physiological parameters are unknown. To achieve this, the migration dynamics of cell clusters should be elucidated.

2. At the cellular level, it is challenging to quantify stem cell migration due to the physical, mechanical, biochemical, and biological complexity of the cellular

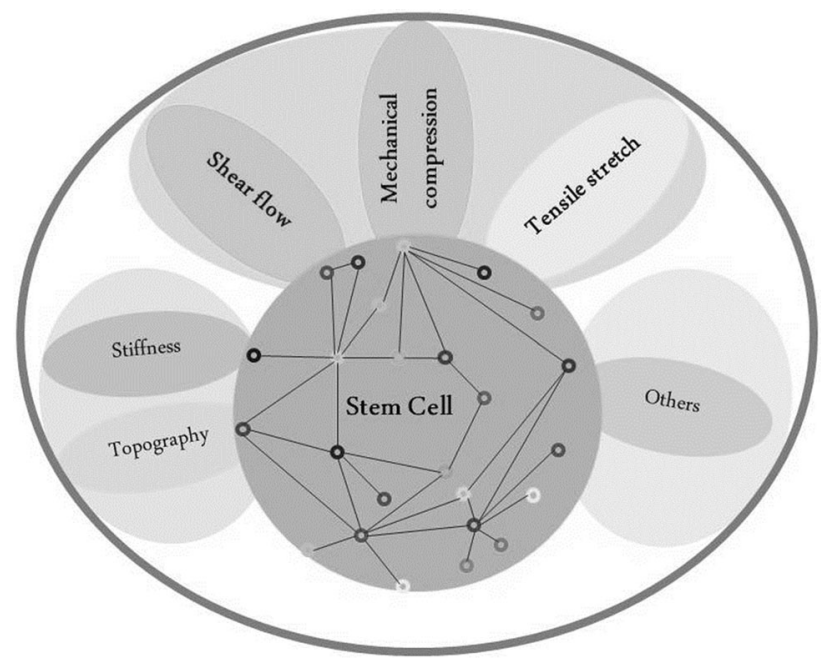

FIGURE 2. Illustration of mechanical microenvironment of stem cell niche. microenvironment. Here, refined modeling of single stem cell migration in various niches is crucial due to understand the diversity of stem cells (e.g. ESC, MSC, HSC, CSC, iPs, and more), the different patterns of mechanical/physical stimuli (i.e. shear flow, tension, compression, stiffness, topology, geometry), various biochemical factors (e.g. concentration, gradient and distribution of chemokine, cytokine, compounds and more), which are hard isolate and examine individually experimentally. Thus, CM will be very helpful to understand the global impact of mechanical and biochemical factors under systematically varied parameter sets.

3. At the intracellular level, it is also critical to elucidate the signaling networks that regulate stem cell migration. Both biochemical and molecular biology data have identified many important signaling molecules in stem cells, but kinetic rates and binding affinity for the each of the different pair of molecules are poorly known; in some cases, it is also unknown how these pairs of molecules are biologically linked. Signaling involved in stem cell differentiation during migration should be taken properly into account; for example, several developmentallyconserved signaling pathways that have emerged as important regulators of stem cell migration need to be further investigated.

4. At the level of a whole organism, whether studying a model animal or human pathophysiology, CM is advantageous in predicting the migration dynamics of stem cells from their origin to their target site. Here large-scale hierarchical modeling is required to map the global features of migrating stem cells where those details for modeling of differentiated cell migration are usually neglected; for example, the role of autocrine and paracrine signaling mechanisms in viability maintenance and efficient self-renewal during embryogenesis. ${ }^{8,15} \mathrm{CM}$ is important also at the level of the organism to generate accurate prognostic estimates from stem cell transplantation.

\section{SUMMARY}

While the regulation of stem cell migration is extensively tested using experimental approaches, their sensitivity and capacity to undergo different patterns of mechanical and biochemical regulators are able to be modeled computationally via theoretical and numerical predictions to mimic their responses in the physiologically $3 \mathrm{D}$ niche. Not only is computational modeling able to help provide the mechanistic interpretation to the measured experimental data, but it also predicts the 
potential key modulators to regulate their migration dynamics. It is also useful in clinical practice such as stem cell therapy, where the questions related to human pathophysiology in health and disease have to be answered. In summary, computational modeling provides new information for stem cell migration in combination with experimental measurements at different levels in such fields as embryonic development, tissue regeneration, and disease therapeutics.

\section{ACKNOWLEDGMENTS}

This work was supported by National Natural Science Foundation of China Grant 31110103918, National Key Basic Research Foundation of China grant 2011CB710904, Strategic Priority Research Program grants XDA01030102 and XDA04020219 and Knowledge Innovation Program Grant 2005-1-16 from Chinese Academy of Sciences (M.L.). MHZ acknowledges the generous support of NIH grant (5U01CA177799).

\section{CONFLICT OF INTEREST}

Xiaofeng Liu, Diego A. Vargas, Dongyuan Lü, Yan Zhang, Muhammad H. Zaman, and Mian Long declare that they have no conflicts of interest.

\section{ETHICAL STANDARDS}

No human or animal studies were carried out by the authors for this article.

\section{REFERENCES}

${ }^{1}$ Aggarwal, S., and M. F. Pittenger. Human mesenchymal stem cells modulate allogeneic immune cell responses. Blood 105:1815-1822, 2005.

${ }^{2}$ Anderson, A. A hybrid mathematical model of solid tumour invasion: the importance of cell adhesion. Math. Med. Biol. 22:163-186, 2005.

${ }^{3}$ Bailon-Plaza, A., and V. D. M. Meulen. A mathematical framework to study the effects of growth factor influences on fracture healing. J. Theor. Biol. 212:191-209, 2001.

${ }^{4}$ Bischofs, I. B., and U. S. Schwarz. Cell organization in soft media due to active mechanosensing. Proc. Natl Acad. Sci. U.S.A. 100:9274-9279, 2003.

${ }^{5}$ Buske, P., J. Galle, N. Barker, G. Aust, H. Clevers, and M. Loeffler. A comprehensive model of the spatio-temporal stem cell and tissue organisation in the intestinal crypt. PLoS Comput. Biol. 7:e1001045, 2011.

${ }^{6}$ Carter, S. B. Haptotaxis and the mechanism of cell motility. Nature 213:256-260, 1967.

${ }^{7}$ Chakraborty, A., R. K. Yadav, M. Liu, M. Tataw, K. Mkrtchyan, A. R. Chowdhury, and G. V. Reddy. Computational tools for quantitative analysis of cell growth patterns and morphogenesis in actively developing plant stem cell niches. Methods Mol. Biol. 876:217-227, 2012.

${ }^{8}$ Consolo, F., C. Bariani, A. Mantalaris, F. Montevecchi, A. Redaelli, and U. Morbiducci. Computational modeling for the optimization of a cardiogenic 3D bioprocess of encapsulated embryonic stem cells. Biomech. Model. Mechanobiol. 11:261-277, 2012.

${ }^{9}$ Dawes, A. T., and L. Edelstein-Keshet. Phosphoinositides and Rho proteins spatially regulate actin polymerization to initiate and maintain directed movement in a one-dimensional model of a motile cell. Biophys. J. 92:744-768, 2007.

${ }^{10}$ Deans, R. J., and A. B. Moseley. Mesenchymal stem cells: biology and potential clinical uses. Exp. Hematol. 28:875884, 2000.

${ }^{11}$ Dimilla, P. A., K. Barbee, and D. A. Lauffenburger. Mathematical-model for the effects of adhesion and mechanics on cell-migration speed. Biophys. J. 60:15-37, 1991.

${ }^{12}$ Drasdo, D., and S. Hohme. A single-cell-based model of tumor growth in vitro: monolayers and spheroids. Phys. Biol. 2:133-147, 2005.

${ }^{13}$ Efroni, S., D. Harel, and L. R. Cohen. Toward rigorous comprehension of biological complexity: modeling, execution, and visualization of thymic T-cell maturation. Genome Res. 13:2485-2497, 2003.

${ }^{14}$ Eke, I., and N. Cordes. Radiobiology goes 3D: how ECM and cell morphology impact on cell survival after irradiation. Radiother. Oncol. 99:271-278, 2011.

${ }^{15}$ Ellison, D., A. Munden, and A. Levchenko. Computational model and microfluidic platform for the investigation of paracrine and autocrine signaling in mouse embryonic stem cells. Mol. Biosyst. 5:1004-1012, 2009.

${ }^{16}$ Enderling, H., L. Hlatky, and P. Hahnfeldt. Tumor morphological evolution: directed migration and gain and loss of the self-metastatic phenotype. Biol. Direct. 5:23, 2010.

${ }^{17}$ Fallica, B., J. S. Maffei, S. Villa, G. Makin, and M. Zaman. Alteration of cellular behavior and response to PI3K pathway inhibition by culture in $3 \mathrm{D}$ collagen gels. PLoS One 7:e48024, 2012.

${ }^{18}$ Fox, J. M., G. Chamberlain, B. A. Ashton, and J. Middleton. Recent advances into the understanding of mesenchymal stem cell trafficking. Brit. J. Haematol. 137:491502, 2007.

${ }^{19}$ Friedl, P., and D. Gilmour. Collective cell migration in morphogenesis, regeneration and cancer. Nat. Rev. Mol. Cell. Biol. 10:445-457, 2009.

${ }^{20}$ Friedl, P., P. B. Noble, P. A. Walton, D. W. Laird, P. J. Chauvin, R. J. Tabah, M. Black, and K. S. Zanker. Migration of coordinated cell clusters in mesenchymal and epithelial cancer explants in vitro. Cancer Res. 55:45574560, 1995.

${ }^{21}$ Geris, L., J. V. Sloten, and H. V. Oosterwyck. Connecting biology and mechanics in fracture healing: an integrated mathematical modeling framework for the study of nonunions. Biomech. Model. Mechanobiol. 9:713-724, 2010.

${ }^{22}$ Gerisch, A., and M. Chaplain. Mathematical modelling of cancer cell invasion of tissue: local and non-local models and the effect of adhesion. J. Theor. Biol. 250:684-704, 2008.

${ }^{23}$ Harjanto, D., J. S. Maffei, and M. H. Zaman. Quantitative analysis of the effect of cancer invasiveness and collagen concentration on 3D matrix remodeling. PLoS One 6:e24891, 2011.

${ }^{24}$ Harjanto, D., and M. H. Zaman. Modleing extracellular matrix reorganization in $3 \mathrm{D}$ environments. PLoS One 8:e52509, 2013. 
${ }^{25}$ Harrison, N. C., R. D. D. Corral, and B. Vasiev. Coordination of cell differentiation and migration in mathematical models of caudal embryonic axis extension. PLoS One 6:e22700, 2011.

${ }^{26}$ Hill, W. D., D. C. Hess, A. Martin-Studdard, J. J. Carothers, J. Q. Zheng, D. Hale, M. Maeda, S. C. Fagan, J. E. Carroll, and S. J. Conway. SDF-1 (CXCL12) is upregulated in the ischemic penumbra following stroke: association with bone marrow cell homing to injury. J. Neuropath. Exp. Neur. 63:84-96, 2004.

${ }^{27}$ Hoffmann, M., J. P. Kuska, M. Zscharnack, M. Loeffler, and J. Galle. Spatial organization of mesenchymal stem cells in vitro-results from a new individual cell-based model with podia. PLoS One 6:e21960, 2011.

${ }^{28}$ Jiang, M. X., S. S. Jin, Z. X. Yao, Q. X. Xiong, and D. Z. Feng. Proportional production research for machiningcenter based on multi-agent. 2007 IEEE International Conference on Automation and Logistics, Vols. 1-6, pp. 2056-2060, 2007.

${ }^{29}$ Jiao, Y., and S. Torquato. Emergent behaviors from a cellular automaton model for invasive tumor growth in heterogeneous microenvironments. PLoS Comput. Biol. 7:e1002314, 2011

${ }^{30}$ Khayyeri, H., S. Checa, M. Tagil, and P. J. Prendergast. Corroboration of mechanobiological simulations of tissue differentiation in an in vivo bone chamber using a latticemodeling approach. J. Orthop. Res. 27:1659-1666, 2009.

${ }^{31}$ Koh, S. H., K. S. Kim, M. R. Choi, K. H. Jung, K. S. Park, Y. G. Chai, W. Roh, S. J. HwangJ, H. J. Ko, Y. M. Huh, H. T. Kim, and S. H. Kim. Implantation of human umbilical cord-derived mesenchymal stem cells as a neuroprotective therapy for ischemic stroke in rats. Brain. Res. 1229:233-248, 2008.

${ }^{32}$ Kolega, J. The movement of cell clusters in vitro: morphology and directionality. J. Cell. Sci. 49:15-32, 1981.

${ }^{33}$ Kotobuki, N., M. Hirose, Y. Takakura, and H. Ohgushi. Cultured autologous human cells for hard tissue regeneration: preparation and characterization of mesenchymal stem cells from bone marrow. Artif. Organs. 28:33-39, 2004.

${ }^{34}$ Lauffenburger, D. A., and A. F. Horwitz. Cell migration: a physically integrated molecular process. Cell 84:359-369, 1996.

${ }^{35}$ Lee, R. H., M. J. Seo, A. A. Pulin, C. A. Gregory, J. Ylostalo, and D. J. Prockop. The CD34-like protein PODXL and alpha 6-integrin (CD49f) identify early progenitor MSCs with increased clonogenicity and migration to infarcted heart in mice. Blood 113:816-826, 2009.

${ }^{36}$ Leung, A. Y. H., and Y. L. Kwong. Haematopoietic stem cell transplantation: current concepts and novel therapeutic strategies. Brit. Med. Bull. 93:85-103, 2010.

${ }^{37}$ Li, Z., Y. Gong, S. Sun, Y. Du, D. Lü, X. Liu, and M. Long. Differential regulation of stiffness, topography, and dimension of substrates in rat mesenchymal stem cells. Biomaterials 34:7616-7625, 2013.

${ }^{38}$ Liu, A. R., L. Liu, S. Chen, Y. Yang, H. J. Zhao, L. Liu, F. M. Guo, X. M. Lu, and H. B. Qiu. Activation of canonical wnt pathway promotes differentiation of mouse bone marrow-derived MSCs into type II alveolar epithelial cells, confers resistance to oxidative stress, and promotes their migration to injured lung tissue in vitro. J. Cell. Physiol. 228:1270-1283, 2013.

${ }^{39}$ Lo, C. M., H. B. Wang, M. Dembo, and Y. L. Wang. Cell movement is guided by the rigidity of the substrate. Biophys. J. 79:144-152, 2000.
${ }^{40}$ Lü, D. Y., X. F. Liu, Y. X. Gao, B. Huo, Y. Y. Kang, J. Chen, S. J. Sun, L. Chen, X. D. Luo, and M. Long. Asymmetric migration of human keratinocytes under mechanical stretch and cocultured fibroblasts in a wound repair model. PLoS One 8:e74563, 2013.

${ }^{41}$ Lü, D., C. Luo, C. Zhang, Z. Li, and M. Long. Differential regulation of morphology and stemness of mouse embryonic stem cells by substrate stiffness and topography. Biomaterials 35:3945-3955, 2014.

${ }^{42}$ Onda, T., O. Honmou, K. Harada, K. Houkin, H. Hamada, and J. D. Kocsis. Therapeutic benefits by human mesenchymal stem cells (hMSCs) and Ang-1 gene-modified hMSCs after cerebral ischemia. J. Cereb. Blood Flow Metab. 28:329-340, 2008.

${ }^{43}$ Painter, K. J. Modelling cell migration strategies in the extracellular matrix. J. Math. Biol. 58:511-543, 2009.

${ }^{44}$ Polacheck, W. J., J. L. Charest, and R. D. Kamm. Interstitial flow influences direction of tumor cell migration through competing mechanisms. Proc. Natl Acad. Sci. U.S.A. 108:11115-11120, 2011.

${ }^{45}$ Ratajczak, M. Z., M. Kucia, J. Ratajczak, and E. K. ZubaSurma. A multi-instrumental approach to identify and purify very small embryonic like stem cells (VSELs) from adult tissues. Micron 40:386-393, 2009.

${ }^{46}$ Roeder, I. Quantitative stem cell biology: computational studies in the hematopoietic system. Curr. Opin. Hematol. 13:222-228, 2006.

${ }^{47}$ Sahin, A. O., and M. Buitenhuis. Molecular mechanisms underlying adhesion and migration of hematopoietic stem cells. Cell Adhes. Migr. 6:39-48, 2012.

${ }^{48}$ Saint-Marcoux, F., B. Royer, J. Debord, F. Larosa, F. Legrand, E. Deconinck, J. P. Kantelip, and P. Marquet. Pharmacokinetic modelling and development of dayesian estimators for therapeutic drug monitoring of mycophenolate mofetil in reduced-intensity haematopoietic stem cell transplantation. Clin. Pharmacokinet. 48:667675, 2009

${ }^{49}$ Sakamoto, Y., S. Prudhomme, and M. H. Zaman. Viscoelastic gel-strip model for the simulation of migrating cells. Ann. Biomed. Eng. 39: 2735-2749, 2011.

${ }^{50}$ Schaller, G., and M. Meyer-Hermann. Multicellular tumor spheroid in an off-lattice Voronoi-Delaunay cell model. Phys. Rev. E 71:051910, 2005.

${ }^{51}$ Schluter, D. K., I. Ramis-Conde, and M. A. J. Chaplain. Computational modeling of single-cell migration: the leading role of extracellular matrix fibers. Biophys. $J$. 103:1141-1151, 2012.

${ }^{52}$ Schwarz, U. S., and I. B. Bischofs. Physical determinants of cell organization in soft media. Med. Eng. Phys. 27:763$772,2005$.

${ }^{53}$ Scianna, M., L. Preziosi, and K. Wolf. A cellular potts model simulating cell migration on and in matrix environments. Math. Biosci. Eng. 10:235-261, 2013.

${ }^{54}$ Sykova, E., and P. Jendelova. Migration, fate and in vivo imaging of adult stem cells in the CNS. Cell Death Differ. 14:1336-1342, 2007.

${ }^{55}$ Vargas, D. A., and M. H. Zaman. Computational model for migration of a cell cluster in three-dimensional matrices. Ann. Biomed. Eng. 39:2068-2079, 2011.

${ }^{56}$ Wu, K. H., B. Zhou, X. M. Mo, B. Cui, C. T. Yu, S. H. Lu, Z. C. Han, and Y. L. Liu. Therapeutic potential of human umbilical cord-derived stem cells in ischemic diseases. Transpl. Proc. 39:1620-1622, 2007.

${ }^{57}$ Wu, K. H., B. Zhou, C. T. Yu, B. Cui, S. H. Lu, Z. C. Han, and Y. L. Liu. Therapeutic potential of human umbilical 
cord derived stem cells in a rat myocardial infarction model. Ann. Thorac. Surg. 83:1491-1500, 2007.

${ }^{58}$ Zaman, M. H., R. D. Kamm, P. Matsudaira, and D. A. Lauffenburger. Computational model for cell migration in three-dimensional matrices. Biophys. J. 89:1389-1397, 2005.
${ }^{59}$ Zepeda-Moreno, A., R. Saffrich, T. Walenda, V. T. Hoang, P. Wuchter, S. Sanchez-Enriquez, A. Corona-Rivera, W. Wagner, and A. D. Ho. Modeling SDF-1-induced mobilization in leukemia cell lines. Exp. Hematol. 40:666-674, 2012. 